\title{
Synthetic Spectra of the Type Ia SN 2002bo
}

\author{
M. Stehle ${ }^{1,2}$ and P.A. Mazzali ${ }^{3,1}$
}

1 Max-Planck-Institut für Astrophysik, P.O. Box 131785741 Garching, Germany; mstehle@mpa-garching .mpg.de

2 Universitäts-Sternwarte München, Scheinerstr. 1, 81679 München, Germany

${ }^{3}$ INAF - Osservatorio Astronomico di Trieste, Via Tiepolo, 11, 34131 Trieste, Italy;

mazzali@ts.astro.it

Summary. SN 2002bo, a type Ia supernova in NGC 3190, is the first object of the European Supernova Collaboration (ESC). It was discovered on 09 March 2002 and is one of the earliest observed SNe. An almost complete set of spectra and photometry is available from 13 days before maximum $B$ light until a few days after. An analysis using synthetic spectra is presented, focusing on the controversial issue of the reddening of SN 2002 bo.

\section{Introduction}

Thermonuclear (type Ia) supernovae are believed to originate from the thermonuclear disruption of a white dwarf composed of carbon and oxygen. It is generally accepted that when the degenerate mass reaches the Chandrasekhar limit $\left(1.4 \mathrm{M}_{\odot}\right)$, explosive carbon ignition occurs and burning to nuclear statistical equilibrium ensues, forming mostly radioactive ${ }^{56} \mathrm{Ni}$. This decays to ${ }^{56} \mathrm{Co}$ and hence to ${ }^{56} \mathrm{Fe}$, emitting $\gamma$-rays and positrons which power the observed optical display of the SN. Intermediate-mass nuclei, e.g. ${ }^{28} \mathrm{Si}$, are produced in the outer, lower-density regions. These elements give rise to the typical observed spectra of $\mathrm{SNe} \mathrm{Ia}$, which are dominated by lines of $\mathrm{Fe}, \mathrm{Si}$ and $\mathrm{S}$. Improving our understanding of $\mathrm{SNe} \mathrm{Ia}$ is important because they offer insight to astrophysical processes under extreme conditions and are of great use to measure cosmological distances. Theoretical models must be tested and constrained through comparison with observed light curves and spectral evolution. Modeling very early spectra is a powerful tool to determine certain parameters, e.g. the epoch, since parameters change significantly and quickly at that time. They not only yield information about the physical properties of the SN ejecta, such as temperature, chemical composition, etc., but also they can be used to verify observational-based estimates of parameters such as reddening, distance and epoch. Here we focus in particular on the reddening estimate. 


\section{Modeling}

We use a Monte Carlo code originally developed by Abbot and Lucy [1] to treat multi-line transfer in the expanding envelopes of hot stars. This code was further developed and adapted to SNe by Mazzali and Lucy $[3,4,5]$. It uses as input a model of the explosion $(\rho \mathrm{v} . v)$, the emergent luminosity $L$, the epoch $t$ (time since explosion), the estimated velocity of the photosphere $v_{p h}$ and a set of abundances. The SN envelope is divided into a number of shells. Homologous expansion is assumed and the temperature in the various shells is computed assuming radiative equilibrium. The Sobolev approximation is adopted and all the radioactive decay and fast-electron energy is deposited below a sharply-defined radius, the "photosphere" (Schuster-Schwarzschild approximation). This energy is distributed equally among packets, which represent "collective photons". Each packet is characterized by a specific frequency and propagates through the envelope where it can interact with electrons and atoms. Interaction with electrons is treated as scattering, while if a packet is absorbed by a line it is re-emitted in one of the allowed downward transitions. The packet is assigned a new frequency and a random direction and the MC procedure continues until the packet either escapes the ejecta or is absorbed back in the photosphere. Finally, the emergent spectrum is computed using the formal integral [3].

The observations of SN 2002bo are discussed by Benetti et al. in this book. Here, we present and discuss synthetic spectra for two different epochs. In view of the somewhat unusual properties of SN 2002bo (e.g. the high velocity of several lines at early epochs), we computed models for the earliest spectrum (13 days before maximum) to determine whether or not the outer abundances are peculiar. We also computed models for a spectrum observed near maximum light in order to check the consistency of our results. We adopted a distance modulus $\mu=31.67$. The density structure was taken from the W7 model [6], and so were the initial abundances, which are assumed to be uniform above the momentary photosphere. The radius of the "photosphere", the emergent luminosity and the abundances were adjusted within a reasonable range until the best match for each spectrum was achieved.

We consider first the early $(-13 \mathrm{~d})$ spectrum. In order to constrain the epoch and the reddening we computed a grid of models, varying the epoch between 4 and 7 days post-explosion and the reddening $E(B-V)$ between 0.00 and 0.45 . From this grid we derived best-match values of $t=5 \pm 1$ days post-explosion and $E(B-V)=0.30$. Thus the maximum-light spectrum corresponds to an epoch of $18 \pm 1$ days post-explosion. With a higher reddening of $E(B-V)=0.45$, the best (but poorer) match was achieved with an epoch of $6 \pm 1$ days post-explosion. Since the observations indicate a value viz. $E(B-V)=0.43 \pm 0.10$, we conservatively adopt $E(B-V)=0.30 \pm 0.15$, and computed models with both $E(B-V)=0.45$ and 0.30 .

Figure 1 shows this spectrum plus the two models. The best match to the data was obtained using a bolometric luminosity of $\log _{10} L=41.94\left[\mathrm{erg} \mathrm{s}^{-1}\right.$ ] at 


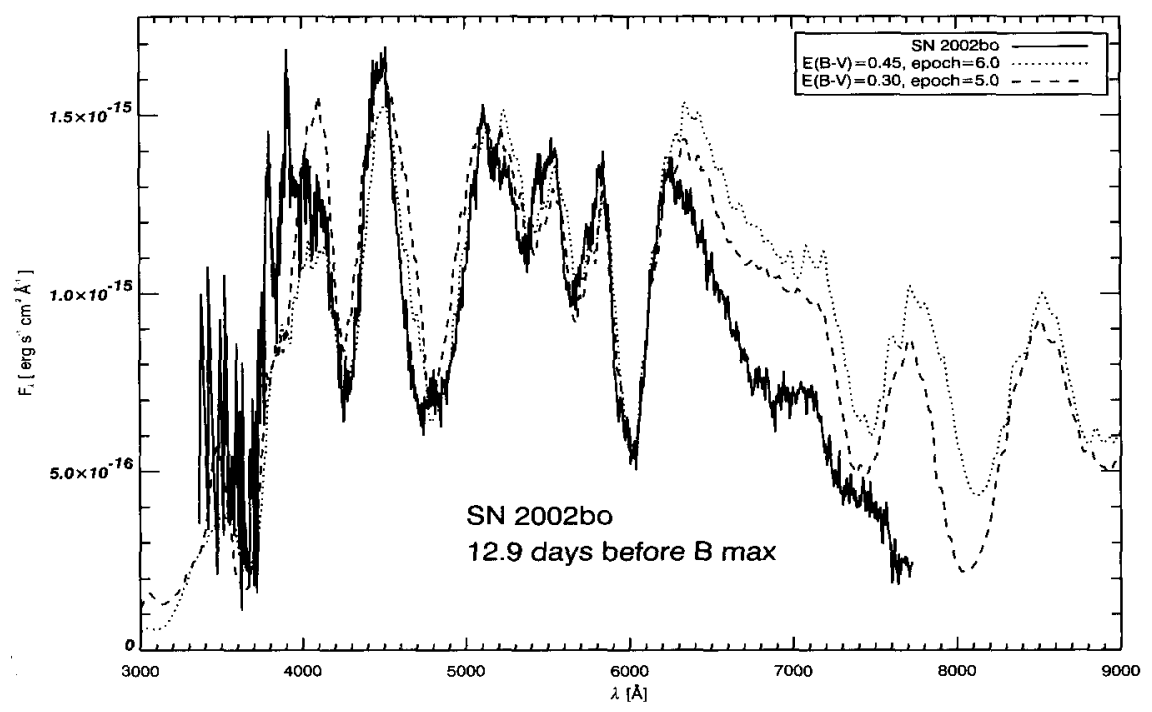

Fig. 1. Spectrum of SN 2002bo 12.9 days before maximum.

a photospheric radius of $v_{p h}=15450 \mathrm{~km} \mathrm{~s}^{-1}$ in the low-reddening (l-r) model. The high-reddening (h-r) model is computed with $\log _{10} L=42.13\left[\mathrm{erg} \mathrm{s}^{-1}\right.$ ] and $v_{p h}=15100 \mathrm{~km} \mathrm{~s}^{-1}$. The luminosity is in good agreement with the observation, which predict $\log _{10} L \approx 42.12\left[\mathrm{erg} \mathrm{s}^{-1}\right]$. These luminosities lead to radiation temperatures at the photospheric radius of $T_{R}=9710 \mathrm{~K}$ (h-r) and $T_{R}=9420 \mathrm{~K}(1-\mathrm{r}) . T_{R}$ is the result of six iterations determining the temperature structure in the ejecta and is influenced by the "backwarming" effect, i.e. photons are scattered back into the photosphere and heat it up. This difference in temperature certainly affects the ionization structure and therefore the emergent spectra significantly. Ca is reduced to 1/10 of its initial value in the h-r model, whilst in the $1-r$ one a reduction to $1 / 4$ is sufficient. All abundances of the iron group elements are significantly lower $(\approx 10 \%$ of the initial values), but here the h-r model needs larger amounts due to the higher temperature. The IME are changed only slightly. The overall reduction of most elements causes a increased $\mathrm{O}$ abundance $(\geq 70 \%)$.

Shortward of $\sim 6500 \AA$ both models reproduce the main features of the data quite well. We examine some of the more prominent features, starting at the shortest wavelengths. We note first that a characteristic of the h-r model is that a higher temperature is required to reproduce the flux in the blue part of the spectrum. This, in turn, tends to increase the ratio of doublyto singly-ionized species. To reproduce the deep MgII $4481 \AA$ absorption at $4300 \AA$, a higher $\mathrm{Mg}$ abundance is required in the h-r higher temperature model to compensate for the smaller fraction of singly-ionized $\mathrm{Mg}$. The deep, broad absorption feature at $\sim 4800 \AA$ is produced mostly by FeII lines. In the h-r model this requires an Fe abundance of 0.02 in the outer parts of 


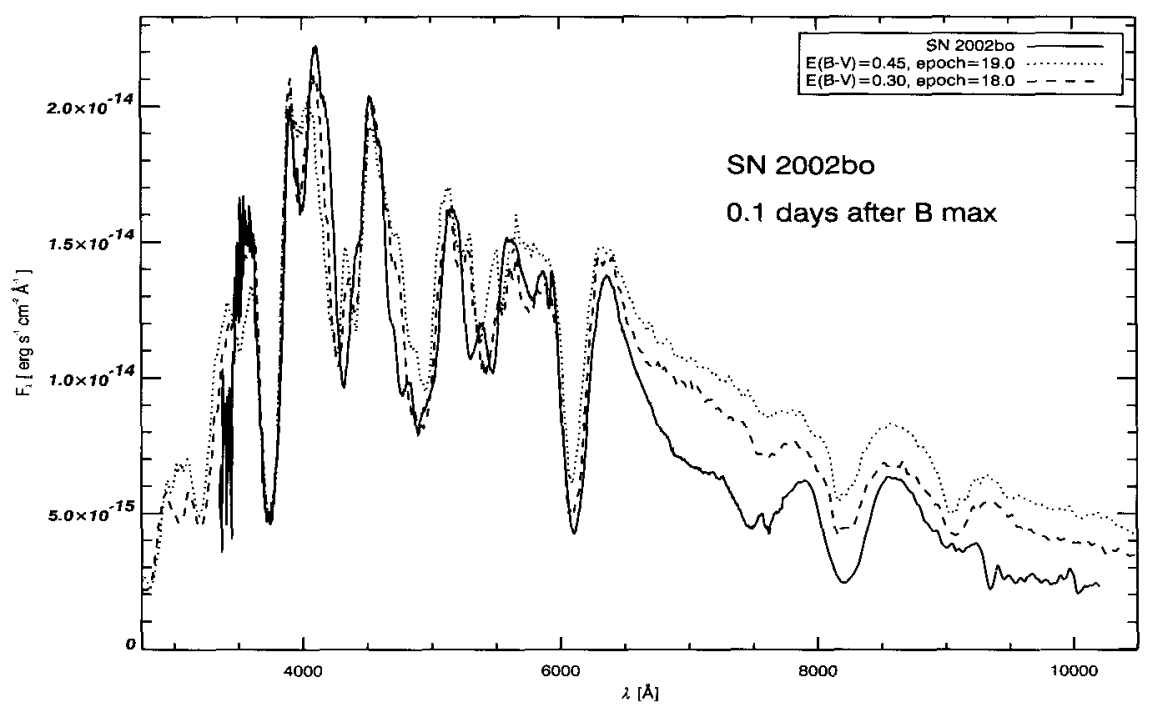

Fig. 2. Spectrum of SN 2002bo 0.1 days after B maximum.

the ejecta, but in the l-r model these drop to 0.015. Thus we see that higher abundances of $\mathrm{Mg}$ and $\mathrm{Fe}$ are required in the h-r model. In contrast, although the deep absorption feature at $\sim 5400 \AA$, attributed to SII $5640 \AA$, is equally well reproduced in both models, we need a somewhat higher $\mathrm{S}$ abundance in the 1-r model (0.05 compared to 0.03$)$. We ascribe this to the smaller fraction of ionized $S$ at this lower temperature compared to the h-r model. However, when we consider Si, very serious difficulties for the h-r model become apparent. We find that in this model, most of the $\mathrm{Si}$ is ionized to SiIII, making it impossible to reproduce the depth of the SiII $5972 \AA$ line. For the l-r model to reproduce the absorption features due to SilI $5972 \AA, 6355 \AA$, a relative abundance of $\mathrm{Si}$ of 0.12 by mass at high velocities $\left(v \geq 15400 \mathrm{~km} \mathrm{~s}^{-1}\right)$ is required. Since $W 7$ predicts no $\mathrm{Si}$ at this velocity this supports the possible explanation of mixing, but the corresponding low-velocity $\mathrm{O}$ is not observed at later epochs. Alternatively, it may suggests that $O$ was at least partially burned to Si even in the outer layers. At longer wavelengths, both models produce a large excess of flux. This is due to the limitation of the SchusterSchwarzschild approximation which is used in the code. In the red part of the spectrum, where line opacity is low, the photosphere actually lies at a greater depth than is estimated in the code, and consequently the model overestimates the flux.

We turn now to the near-maximum spectrum (Fig. 2). The photospheric radius drops to $9750 \mathrm{~km} \mathrm{~s}^{-1}$ for the h-r model and to $9000 \mathrm{~km} \mathrm{~s}^{-1}$ for the l-r one. The photospheric luminosities are $43.27\left[\mathrm{erg} \mathrm{s}^{-1}\right]$ and $43.03\left[\mathrm{erg} \mathrm{s}^{-1}\right]$, respectively. Therefore again the $\mathrm{h}-\mathrm{r}$ model has a higher temperature $\left(T_{R}=\right.$ $13420 \mathrm{~K})$ compared to the $1-\mathrm{r}$ model $\left(T_{R}=12760 \mathrm{~K}\right)$. Both models have 
problems fitting the region around $4300-4500 \AA$, which is mostly dominated by SilI lines. In both cases we need a high Si abundance $(62.1 \%$ for the $\mathrm{h}-\mathrm{r}$ model and $66.7 \%$ for the $1-r$ one, respectively) indicating that the part of the ejecta with velocities near $10,000 \mathrm{~km} \mathrm{~s}^{-1}$ is dominated by IME. As with the earlier epoch, in the h-r model almost all $\mathrm{Si}$ is doubly ionized. While this results in the absorption of the $6355 \AA$ line being slightly too shallow, the most dramatic difficulty is that the $5958 \AA$ line is completely absent. Moreover, this model produces a rather strong SiIII $4567 \AA$ absorption (near $4400 \AA$ ) which is not present in the data at all. These problems are much reduced in the l-r model although the persistence of a weak absorption due to SiIII $4567 \AA$ in the model suggests that $E(B-V)$ could be even smaller than 0.30 . As with the early-epoch spectrum, both models overproduce the flux longward of $\sim 6500 \AA$, with the h-r model being the most discrepant. The observed absorption at $7500 \AA$ might be identified as OI $7771 \AA$. However, even with an unphysically high $\mathrm{O}$ abundance we cannot reproduce this feature since the high temperature ionizes all the neutral $O$. The CaII absorption at $\sim 3750 \AA$ due to the $3933,68 \AA \mathrm{H}$ and $\mathrm{K}$ doublet matches the observation very well, whilst the absorption at $\sim 8200 \AA$ due to the $\sim 8500 \AA$ IR triplet has the correct strength, but it is superposed on a continuum that is too high. The match is somewhat better in the l-r model. In general, we find that the difficulties caused by the high reddening are even larger at this epoch.

We conclude that modeling of both epochs suggests a reddening value $\mathrm{E}(B-V)=0.30$. This is significantly smaller than the value derived from the Lira relation $\left[\mathrm{E}(B-V)_{L}=0.43\right]$. This is indicated by the line ratios, line depths, overall shape of the spectra and the model abundances. Apart from the reddening problem, the abundances and spectral features of the models confirm that SN 2002bo was a normal SN Ia. The model-derived explosion epoch of $18 \pm 1$ days is consistent with the rise-time derived from our photometry using the Riess et al. procedure, and with the average value for SNe Ia given by Riess et al $[7,8]$. It will be very interesting to see how the various elements are distributed throughout the ejecta. These aspects are being investigated and will be discussed in a separate analysis using an improved version of the MC code, including abundance stratification.

\section{References}

1. D.C. Abbott, L.B. Lucy: Astrophys. J. 288, 679 (1985)

2. K. Iwamoto et al. : Astrophys. J. Suppl. 125, 439 (1999)

3. L.B. Lucy: Astron. Astrophys. 345, 211 (1999)

4. P.A. Mazzali, L.B. Lucy: Astron. Astrophys. 279, 447 (1993)

5. P.A. Mazzali: Astron. Astrophys. 363, 705 (2000)

6. K. Nomoto, F. Thielemann, K. Yokoi: Astrophys. J. 286, 644 (1984)

7. A.G. Riess et al.: Astron. J. 116, 1009 (1998)

8. A.G. Riess etal.: Astron. J. 118, 2675 (1999) 